\title{
Prototipo de un Sistema Basado en Localización para dinamizar el turismo en el cantón Azogues
}

Martin Geovanny Zhindon Mora. Luis Cabrera Morales. Sebastián Quevedo Sacoto.

Recibido: febrero 2017 Aprobado: mayo 2017 


\title{
Prototipo de un Sistema Basado en Localización para dinamizar el turismo en el cantón Azogues
}

\section{Prototype of a Location Based Service to revitalize Tourism in the Azogues canton}

\author{
Martin Geovanny Zhindon Mora. Luis Cabrera Morales. Sebastián Quevedo Sacoto. \\ Unidad Académica de Ingeniería, Industria y Construcción \\ Universidad Católica de Cuenca. \\ Av. de las Américas y Tarqui. \\ Cuenca - Ecuador \\ mgzhindonm@ucacue.edu.ec
}

\begin{abstract}
Resumen
El cantón Azogues posee un potencial turístico cultural que no ha sido explotado debido a la falta de recursos para promocionar el turismo. El objetivo de este trabajo es aportar en este ámbito con la implementación de un prototipo de Servicio Basado en Localización (SBL) que permita determinar la ubicación geográfica, eventos a realizarse e información detallada de los lugares turísticos del cantón Azogues. Las tecnologías embebidas en los dispositivos móviles han dado lugar a los SBL, siendo el turismo el principal consumidor de estos servicios. Para llevar a cabo el desarrollo del prototipo se combinó la metodología de Despliegue de la Función de la calidad (QFD, por sus siglas en ingles), para la valoración de la especificación de los requisitos de software (ERS) y para determinar las herramientas de desarrollo que mejor se acoplen a los requerimientos en el ámbito del turismo y la metodología ágil SCRUM para gestionar el proceso de desarrollo del prototipo. Como resultado se implementó un prototipo híbrido multicapa utilizando principalmente el marco de referencia Apache Córdova para el cliente móvil y Java Edición Empresarial (JEE) para el servidor.
\end{abstract}

Palabras clave: turismo, localización, móvil, Apache Córdova, Java

\begin{abstract}
Azogues canton possesses a tourist and cultural potential; however, due to the lack of resources, it has not been exploited to promote tourism. The main objective of this work is to contribute to tourism through the implementation of a Location Based Service (LBS) prototype. This prototype allows determining the geographical location, forthcoming events and detailed information on the different tourist destinations of Azogues Canton. Embedded technologies in the mobile devices have led to the LBS, with tourism as the major consumer of this service. In order to accomplish the development of this prototype, the Quality Function Deployment (QFD) methodology was combined to assess the Software Requirement Specifications (SRS) and to establish the development tools that fit the best with the tourism requirements. In addition, the SCRUM agile methodology was also combined to manage the development process of the prototype. As a result, a multi-layer hybrid prototype was implemented by using mainly the Apache Cordova reference framework for the mobile customer and Java Enterprise Edition (JEE) for the server.
\end{abstract}

KEYWORDS: Tourism, Location, Mobile, Apache Cordova, Java 


\section{Introducción}

El turismo en la actualidad se ha desarrollado de manera que los destinos turísticos experimentan demandas de tipo cultural, tradicional e incluso gastronómica [1] frente a esto las ciudades se ven obligadas a brindar sus servicios empleando formas innovadoras para satisfacer las nuevas demandas de los turistas que buscan experiencias diferentes. El turismo cultural se define como "una forma de turismo alternativo que representa la consumación de la comercialización de la cultura" [2], es decir, fomentar el turismo por medio de la promoción cultural para incrementar las visitas de turistas e ingresos económicos.

En el Ecuador, el turismo cultural ha incrementado su actividad en los últimos años permitiendo posicionar al sector turístico como una de las fuentes principales de ingresos no petroleros del país, los visitantes provienen principalmente de Colombia con un $23,8 \%$ del total de los arribos al país, en segundo lugar, está Estados Unidos con un $16,6 \%$ y finalmente Perú con un $11,5 \%$; ingresando el país alrededor de 1.5 millones de dólares por motivos turísticos [3].

El cantón Azogues posee un potencial turístico [4] debido a la cultura existente en la región. Para explotar este potencial, el Gobierno Autónomo Descentralizado Municipal de Azogues implementó una página web para la publicación de los puntos de turísticos, hospedajes y restaurantes, sin embargo según [5], hacen falta recursos para promocionar el turismo local, debido a esto como medio alternativo se han utilizado las redes sociales para la publicación de los eventos a realizarse dentro del cantón, resultando insuficiente debido a los bajos ingresos que se han registrado en el sector turístico.

Según los datos de las estadísticas de ingresos de turistas al cantón Azogues proporcionadas por la Unidad de Turismo del GAD Municipal (Figura 1), existe una tendencia a la disminución del porcentaje de visitantes que llegan a la ciudad en los días festivos.

=AÑO 2008 AÑO $2009=$ AÑO 2010 AÑO 2011

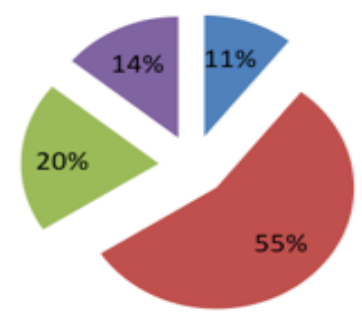

Figura 1. Demanda de visitantes por año.

Fuente: Unidad de Turismo, GAD Municipal del cantón Azogues.
El uso de las Tecnologías de Información y Comunicaciones se ha consolidado como parte fundamental en el proceso de la preparación y ejecución de planes turísticos tales como su promoción, difusión, venta, información, entre otros [6], estas herramientas han significado un desarrollo humanístico y tecnológico en lo que a turismo se refiere, generándose así una disciplina llamada eTourism o turismo electrónico [6] que crea turistas caracterizados por un constante uso de herramientas tecnológicas en el sector turístico [7].

Los avances en la actualidad han permitido que la tecnología sea embebida en dispositivos móviles, esto ha dado lugar a los SBL. Los servicios basados en localización son el resultado de la convergencia de tres tecnologías innovadoras: el internet, el sistema de posicionamiento global (GPS) y las interfaces de usuario, siendo una tecnología que ha evolucionado de manera constante desde finales de los noventa [8].

El avance de la tecnología permite la utilización de los dispositivos móviles como medios para la obtención de información en tiempo real sobre ubicaciones geográficas, clima, tráfico, entre otros [9], y facilitando su comprensión a través de mapas mediante los cuales se presentan puntos de interés para el turista, esto ha permitido que se desarrollen aplicaciones como; aplicaciones de tráfico en tiempo real que proveen información sobre congestionamiento vehicular en las vías mediante la utilización de sistemas de localización y el seguimiento de la ubicación geográfica de los usuarios [10], las redes sociales como Facebook y Twitter también utilizan los servicios de localización de los dispositivos móviles para ubicar amigos cercanos o recomendar al usuario realizar ciertas actividades dependiendo del lugar en donde se encuentre, además existen aplicaciones que permiten encontrar personas con intereses en común o hasta una pareja de acuerdo a un rango de localización, de esta manera se ha demostrado que las aplicaciones de redes sociales usan en gran medida los SBL [11], finalmente otro ejemplo esta en su utilización en el rastreo de transporte público, permitiendo conocer el tiempo estimado del arribo de autobuses, trenes y taxis a un determinado lugar [12].

El internet llevado a los dispositivos móviles ha evolucionado la comunicación de las personas con el medio, y en el ámbito turístico está ayudando a que los usuarios encuentren toda clase de información que conlleva una evolución en el conocimiento de la sociedad, siendo el turismo y la tecnología los sectores que más crecen en la economía global [13]; la unificación del internet y los servicios basados en localización son fundamentales para los usuarios turistas ya que proporcionan información necesaria para la ubicación y localización de puntos de interés. 
El turismo es el principal consumidor de los SBL [14], estos han contribuido al crecimiento del turismo global, ya que facilitan la ubicación geográfica un destino turístico por visitar. Los dispositivos móviles se han convertido en la herramienta idónea para el uso de los SBL gracias a su portabilidad y a su facilidad de uso [15]. La disponibilidad de las herramientas han permiten que sea posible el desarrollo de estos SBL.

Azogues no cuenta con un medio que proporcione información sobre los eventos a realizarse en los lugares turísticos ni tampoco sus ubicaciones geográficas. A pesar de existir aplicaciones como OSMAND $^{1}$ que permite la navegación por medio de mapas usando SBL, estos carecen de información detallada de los puntos de interés del cantón, así como de eventos a realizarse.

El objetivo de este trabajo es implementar un prototipo SBL para dispositivos móviles que permita determinar la ubicación geográfica, eventos a realizarse y proporcione información detallada de los lugares turísticos del cantón Azogues.

\section{Materiales y Métodos}

En el desarrollo del presente trabajo se combinaron las metodologías QFD y SCRUM. La metodología QFD fue utilizada para la recopilación de los requisitos de software y el establecimiento de las herramientas capaces de satisfacer los requisitos, mientras que la metodología Scrumb fue utilizada para gestionar el proceso de desarrollo del prototipo.

\subsection{Metodología QFD}

La metodología QFD la define [16] como "un sistema para trasladar los requerimientos del usuario en requerimientos apropiados para cada fase desde la investigación y desarrollo hasta la ingeniería y la implementación”. El motivo de aplicar la metodología QFD en este trabajo es iniciar la fase de desarrollo del prototipo con una lista concreta de pasos a seguir tanto en los requerimientos del usuario como en las herramientas necesarias para cubrir los requerimientos. La estructura de ésta metodología se presenta en varias etapas que inician desde la recolección de los requerimientos del usuario sobre el desarrollo del prototipo y finaliza con la relación entre las herramientas utilizadas y el análisis, para determinar el funcionamiento del desarrollo cuyas etapas se definen de la siguiente manera según [17];

La primera etapa dentro de la metodología QFD fue determinar los requisitos de software los cuales se

\footnotetext{
${ }^{1}$ http://osmand.net/, Direcciones de navegación automatizadas de Open Street Maps.
}

establecieron mediante la aplicación de encuestas a una muestra de la población del cantón Azogues. El tamaño de la muestra se fijó de acuerdo a la siguiente fórmula [18]:

$$
n=\frac{N * Z^{2} * p *(1-p)}{(N-1) * e^{2}+Z^{2} * p *(1-p)}
$$

Donde buscamos un nivel de confianza del $95 \%$ por lo que la desviación del valor medio aceptado es $\mathrm{Z}=1.96$. El tamaños de nuestro universo correspondiente a la población de Azogues es $\mathrm{N}=70064$. El error admitido fue del $5 \%$ a igual que la proporción por lo que e $=0.05$ y $\mathrm{p}=0.05$ respectivamente. Luego de aplicar esta fórmula el número total de encuestados fue 76.

Las preguntas de las encuestas consistieron en valorar en un rango del uno al cinco la importancia de cada uno de los requisitos de software, siendo uno irrelevante y cinco muy importante, cada requisito se software valorado se convirtió en las entradas de los QUE de la matriz [19].

La segunda etapa consistió en definir los COMO que son un conjunto de herramientas potenciales para el desarrollo del prototipo para satisfacer los requisitos de software. En la tercera etapa se relacionaron los requerimientos de software y las herramientas por medio de valores que muestran el nivel de relación entre ellas usando tres niveles representativos que son uno para relación baja, dos para relación media, y tres para relación fuerte. En la cuarta etapa se realizó una comparación con un producto desarrollo al que se va a ofrecer, mediante un rango que representa la valoración del presente desarrollo en relación con un desarrollo similar para obtener los requerimientos a mejorar, en este caso se hizo la comparación con Google $\mathrm{Maps}^{2}$ y OSMAND.

En la quinta etapa se promedió la comparación del presente desarrollo con un desarrollo similar obteniendo un valor denominado "objetivo a alcanzar" que permite realizar el prototipo de manera que se cumplan los requerimientos del usuario, ofreciendo un producto de calidad a los usuarios y así satisfacer sus necesidades. En la sexta etapa se obtuvieron las ponderaciones tanto absolutas como relativas por medio del análisis de los datos recopilados en las etapas de evaluación de la competencia y los objetivos del proceso, dando así a conocer el grado de concentración del desarrollo en cada requerimiento del usuario. Las ponderaciones son el nivel de importancia que se otorgan en el desarrollo del producto a los requerimientos iniciales.

${ }^{2}$ http://whatis.techtarget.com/definition/Google-Maps, Servicio Web que con información acerca de regiones geográficas del mundo. 
En la séptima etapa se analizaron las actividades o herramientas que se utilizaron para el desarrollo del prototipo realizando el cálculo de la importancia técnica que es un valor que se asigna a cada CÓMO del diagrama, representando una comparación técnica entre la presente propuesta y un desarrollo similar. Finalmente, en la etapa ocho se elabora la matriz de correlaciones mediante el análisis de los COMO, que consiste en verificar las relaciones de cada herramienta entre sí y sirve para reflejar su importancia en el desarrollo del prototipo [19].

De esta manera, mediante el análisis QFD se obtuvieron los resultados de los requisitos de software más importantes y las herramientas adecuadas a utilizar en la implementación del prototipo para satisfacer los requisitos. (Figura 2)

\begin{tabular}{|c|c|c|c|c|c|c|c|}
\hline & 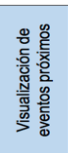 & 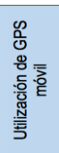 & 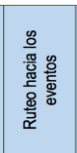 & 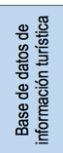 & 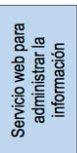 & 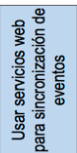 & 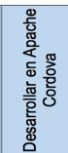 \\
\hline Conocer eventos a realizarse & 9 & 3 & 3 & 9 & 3 & 1 & 1 \\
\hline $\begin{array}{l}\text { Saber la ubicación de los } \\
\text { eventos }\end{array}$ & 9 & 9 & 9 & 9 & 3 & 3 & 1 \\
\hline $\begin{array}{l}\text { Conocer fecha y hora de los } \\
\text { eventos }\end{array}$ & 9 & 1 & 1 & 9 & 3 & 3 & 1 \\
\hline Cómo llegar al evento & 3 & 9 & 9 & 1 & 1 & 3 & 1 \\
\hline $\begin{array}{l}\text { Tener información acerca de los } \\
\text { puntos de interés turisticos del } \\
\text { canton }\end{array}$ & 1 & 1 & 3 & 9 & 9 & 9 & 1 \\
\hline $\begin{array}{l}\text { Mantener información } \\
\text { actualizada }\end{array}$ & 3 & 1 & 1 & 3 & 9 & 9 & 3 \\
\hline $\begin{array}{l}\text { Aplicación compatible con } \\
\text { distintos smartphones }\end{array}$ & 1 & 3 & 1 & 1 & 1 & 3 & 9 \\
\hline $\begin{array}{l}\text { Conocer sobre el clima del } \\
\text { evento }\end{array}$ & 1 & 1 & 1 & 1 & 1 & 1 & 1 \\
\hline $\begin{array}{l}\text { Conocer sobre la seguridad del } \\
\text { evento }\end{array}$ & 1 & 1 & 1 & 1 & 1 & 1 & 1 \\
\hline $\begin{array}{l}\text { Orientación deseada } \\
\text { Ponderación absoluta }\end{array}$ & $\begin{array}{c}\uparrow \uparrow \\
459,32\end{array}$ & $\frac{\uparrow}{351,14}$ & \begin{tabular}{|c|}
$\uparrow$ \\
348,97
\end{tabular} & $\begin{array}{c}\uparrow \\
522,98\end{array}$ & \begin{tabular}{c|}
$\uparrow$ \\
339,82
\end{tabular} & \begin{tabular}{|c|}
$\uparrow$ \\
362,66 \\
\end{tabular} & $\begin{array}{c}\uparrow \\
205,73 \\
\end{array}$ \\
\hline Ponderación relativa (\%) & 17,73 & 13,55 & 13,47 & 20,19 & 13,12 & 14,00 & 7,94 \\
\hline Orden de importancia & 6 & 4 & 3 & 7 & 2 & 5 & 1 \\
\hline Valoración técnica & BDEv & GPS & Mapzen & BD & WebSvc & Internet & Cordove \\
\hline Mi pro & 4 & 1 & 1 & 1 & 1 & 1 & 1 \\
\hline OSMAI & 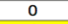 & 1 & 1 & 0 & 0 & 1 & 0 \\
\hline Objetivo técnico & 1 & 1 & 1 & 1 & 1 & 1 & 1 \\
\hline
\end{tabular}

Figura 2. Matriz QFD

\subsection{Metodología Scrum}

La metodología Scrum se basa en un proceso iterativo e incremental (Figura 3), en el cual cada una de sus iteraciones se define por los requisitos y el resultado de cada iteración es un producto incremental. Durante el desarrollo de cada iteración existe un proceso de inspección y adaptación constante mediante el cual se asegura que la funcionalidad se encuentre acorde a los requisitos de software. Este proceso se repite hasta que el desarrollo de software concluya [20]

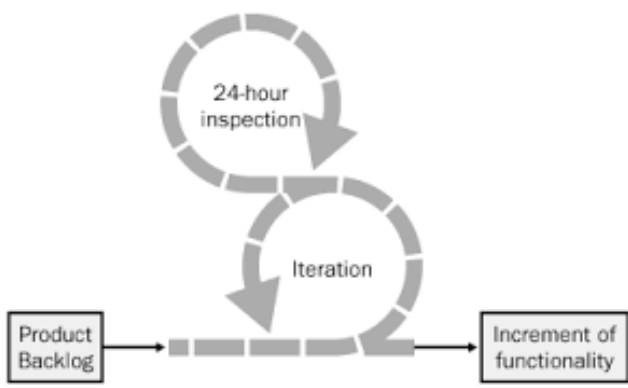

Figura 3. Esqueleto Scrum

Fuente: Agile Project Management with Scrum, 2
Los requisitos con los cuales se definieron las iteraciones en el desarrollo de este prototipo fueron los obtenidos como resultado de la aplicación de la metodología QFD.

Al inicio de cada iteración se estableció que es lo que se tiene que hacer en base a los requisitos para los cuales es posible obtener un incremento y se evaluaron las herramientas aptas de acuerdo a la metodología QFD y se determinó como construir la funcionalidad. Al final de la iteración se evaluó el incremento y se volvió a repetir este proceso hasta implementar todas las funcionalidades requeridas para el prototipo.

El detalle de las herramientas utilizadas para el desarrollo del prototipo de acuerdo con el análisis realizado con la metodología QFD se presenta a continuación:

Apache Cordova es un marco de desarrollo móvil libre y de código abierto que permite crear aplicaciones nativas multiplataforma usando tecnologías web estándar como HTML5 [21]. Maven es "una herramienta de software para la gestión y construcción de proyectos Java" [22]. Ionic Es un Kit de Desarrollo de Software (SDK) de código abierto que fue construido basado en AngularJS y Apache Cordova. Provee herramientas y servicios para desarrollar aplicaciones móviles híbridas utilizando tecnología web como HTML, CSS y JavaScript [23]. AngularJS es un marco de trabajo de JavaScript que extiende los atributos de HTML mediante Directivas y une los datos HTML mediante Direcciones [24].

JBoss es un servidor de aplicaciones de código abierto implementado en Java y puede ser utilizado en cualquier sistema operativo de escritorio [25]. RichFaces es una biblioteca de código abierto que se ejecuta mediante el servidor JBoss que brinda interfaces gráficas para las aplicaciones desarrolladas con tecnología web [26]. Mapzen es una plataforma de mapeado de código abierto, sustentable y accesible que permite mostrar, buscar, navegar y rutear mapas mediante servicios provistos por los desarrolladores de su comunidad [27]. Leaflet es una librería JavaScript de código abierto que permite el desarrollo de mapas interactivos utilizando tecnología web que soporta tanto plataformas móviles como de escritorio [28].

Los componentes principalmente utilizados en el desarrollo son; Java Server Faces que es una especificación para crear interfaces de usuario basadas en componentes para aplicaciones web, Java Persistence API que es una especificación de la interfaz de programación de aplicaciones JAVA que describe la gestión de los datos relacionales en los desarrollos y Enterprise JavaBeans que es un componente que encapsula la lógica de negocio del prototipo [29]. 


\section{Resultados}

\subsection{QFD}

Los resultados de la aplicación de la metodología QFD permiten ver en porcentajes (Figura 4) la importancia de cada uno de los requerimientos no funcionales de la aplicación. Con base en estos resultados se implementaron los requisitos de mayor importancia es decir los que presentaron porcentajes altos y se descartaron los requerimientos de clima y seguridad, que fueron los de menor relevancia, con el $5 \%$ y $8 \%$ respectivamente.

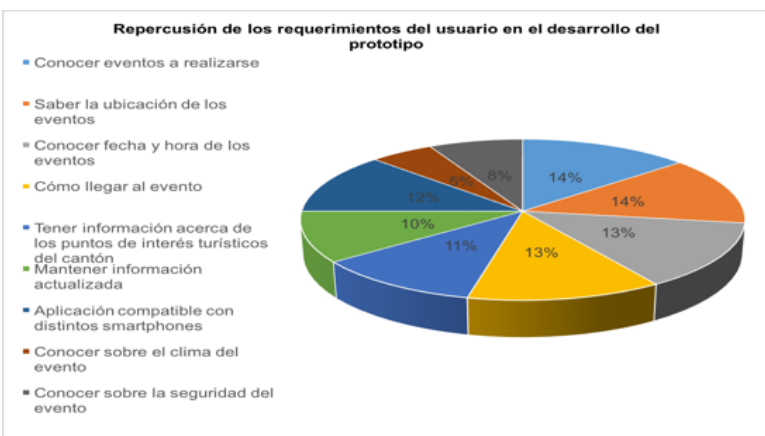

Figura 4. Prioridad de implementar requerimientos

En la (Figura 5) se muestra el porcentaje con el cual aportan las herramientas seleccionadas en la implementación de cada uno de los requerimientos no funcionales.

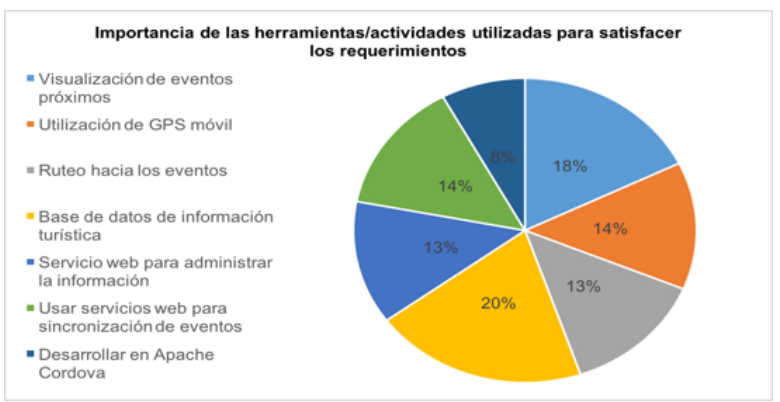

Figura 5. Aporte funcional de las herramientas de desarrollo

\subsection{El prototipo}

El SBL fue implementado en función de los resultados obtenidos de aplicar la metodología QFD, las herramientas como los requerimientos corresponden a los que mayor puntaje obtuvieron en la implementación de la matriz QFD. El SBL consta de dos componentes principales, el cliente que corresponde a la aplicación móvil con la cual interactúa el usuario turista y el sistema de administración que es el servidor.
La arquitectura del cliente se encuentra establecida de acuerdo al marco de desarrollo Apache Cordova (Figura 6), el cual accede al GPS del dispositivo móvil con sistema operativo Android mediante el uso de conectores JavaScript y accede a la información mediante el consumo de un servicio web implementado en el servidor.

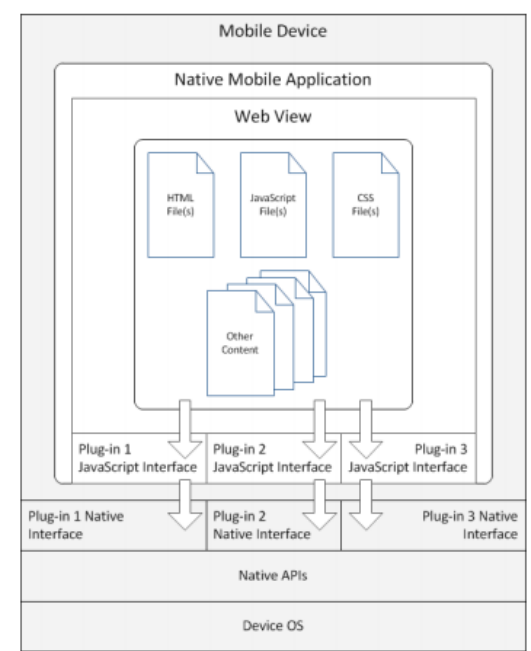

Figura 6. Arquitectura del Cliente

Fuente: Apache Cordova 3 Programming

El cliente dispone de la Interfaz de Usuario Web (WUI, por sus siglas en inglés) principal que integra el acceso a todas las funciones disponibles. Desde esta WUI el turista puede acceder a una guía categorizada con información de los sitios turísticos del cantón, acceder a una lista de eventos programados a desarrollarse en cada uno de los sitios turísticos y acceder a un mapa para visualizar la distribución geográfica de los eventos. Además la WUI principal presenta de forma permanente los eventos próximos a realizarse (Figura 7).

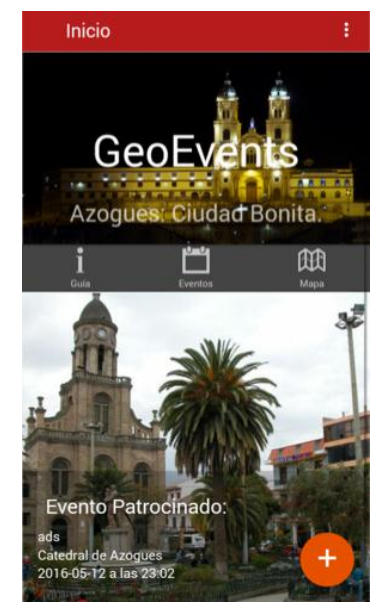

Figura 7. WUI Principal 
El propósito de la WUI de categorías es mostrar al turista los lugares turísticos de forma organizada para que de acuerdo a sus preferencias ubique fácilmente los lugares que son de su interés. De igual manera la WUI Presenta al turista los eventos a desarrollarse en cada uno de los lugares incluidos la fecha y hora del mismo, además muestra la distancia a la que se encuentra el evento de su posición actual. La WUI de mapa muestra la distribución geográfica de los lugares turísticos a través de un mapa, además permite obtener la ruta más corta desde la ubicación actual del turista hacia el lugar de interés (Figura 8).

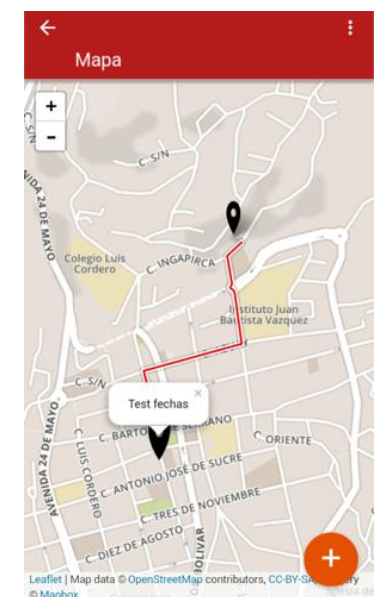

Figura 8. WUI Mapa de lugares turísticos

El desarrollo del administrador de prototipo se realizó con JEE6, en la capa de presentación se utilizaron las librerías JSF y RichFaces; en la capa del servicio se utilizó EJB y en la capa de persistencia se utilizó JPA, encargado de interactuar con el gestor de base de datos PostgreSQL. El componente de administración permite realizar las operaciones de creación, consulta, actualización y eliminación de categorías, lugares turísticos y eventos. Esto permite que el sistema se ajuste a la dinámica existente en las actividades relacionadas con el turismo (Figura 9).

\section{Referencias}

[1] D. Rypkema, C. Cheong y R. Mason, «Measuring Economic Impacts of Historic Preservation,» noviembre 2011.

[2] T. Cultural y C. Turísticas, «Horizontes Antropológicos,» UFRGS. IFCH. PPGAS, vol. 9, pp. 31-58, 2003.

[3] Ministerio de Turismo, «Resumen 2014: Turismo ecuatoriano, en su mejor momento,» 3012 2014. [En línea]. Available: http://www.turismo.gob.ec/resumen2014-turismo-ecuatoriano-en-su-mejor-momento/.

[Último acceso: 2017].

[4] K. Farfán, Interviewee, Planes para el fortalecimiento turístico de Azogues. [Entrevista]. 13 022016.

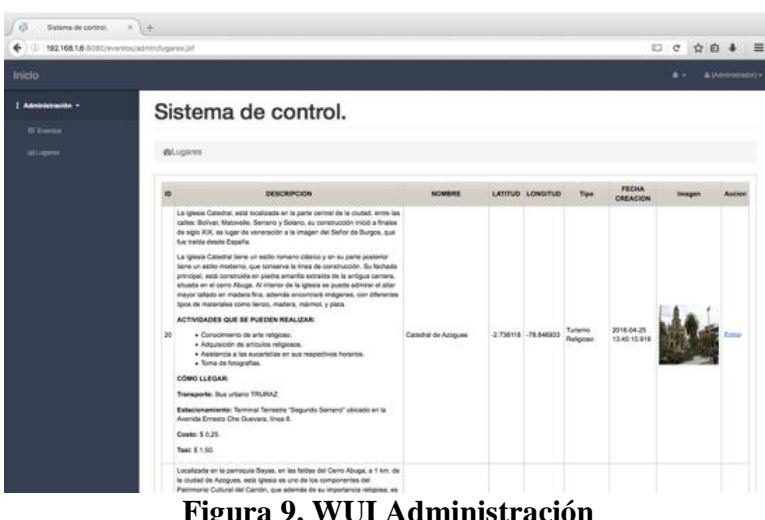

Figura 9. WUI Administración

\section{Conclusiones}

El prototipo desarrollado proporciona información detallada de los lugares turísticos del cantón Azogues así como su ubicación geográfica y de los eventos a realizarse en estos. La combinación de las metodologías QFD y SCRUM permitió establecer de forma clara y directa los requisitos de software y las herramientas para implementar la funcionalidad de cada uno de los requisitos.

La disponibilidad de herramientas gratuitas como el marco de desarrollo Apache Cordova y Java Edición empresarial permitieron que sea posible la implementación de este prototipo que es un aporte alternativo para la falta de promoción del turismo en el cantón Azogues.

Como trabajos futuros se plantea; elaborar un plan para la puesta en producción de prototipo y la creación de indicadores que permitan medir su aporte en el sector turístico y la incorporación incremental de nuevos requisitos de software analizados con la metodología QFD e introducidos al SBL mediante Scrum.

[5] D. Araujo, Interview, Falta de recursos para promocionar el turismo en Azogues. [Entrevista]. 2810 2015.

[6] D. Buhalis y R. Law, «Progress in information technology and tourism management: 20 years on and 10 years after the Internet-The state of eTourism research.,» Elsevier Ltd., Dorset, 2012.

[7] R. Chamorro, «Una revolución en el turismo gracias a las TIC,» Oficina de Turismo, Madrid, 2008.

[8] Navipedia contributors, «Navipedia,» 18092014. [En línea]. Available: http://www.navipedia.net/index.php?title=Location_B ased_Services\&oldid $=13187$. [Último acceso: 2402 2016].

[9] R. Kokku, K. Sundaresan y G. Jiang, «Enabling Location Specific Real-time Mobile Applications,» NEC Laboratories America, Princeton, 2008. 
[10] A. Bacchus, B. Hellinga y P. Izadpanah, «An opportunity assessment of wireless monitoring of network-wide road traffic conditions.,» Departamento Ingeniería Civil Universidad Waterloo, Waterloo, 2008. [11] E. Gorrita, R. Sierra y S. Montejo, «Aplicaciones y servicios basados en localización,» Revista Cubana de Ingeniería, La Habana, 2012.

[12] Moovit, «Navigate your city,» 2015.

[13] Y. Stamboulis y P. Skayannis, «Innovation strategies and technology for experience-based tourism.,» Tourism management, 2003.

[14] Enterprise Mobility Xperts, «Location Based Services - Market and trend analysis,» EMX, 2013.

[15] S. K. Thokala, P. Koundinyaa, S. Mishra y L. Shi, «Virtual GPS: a middleware for power efficient localization of smartphones using cross layer approach.,» ACM, New York, 2014.

[16] C. Madu, House of Quality in a Minute: Quality Function Deployment, vol. 2, New York: Department of Management and Management Science, Lubin School of Business, 2006, p. 1.

[17] J. Revelle, J. Moran y C. Cox, The QFD Handbook, New York: John Wiley \& Sons, 1998.

[18] C. Ochoa, «netquest,» 11 Noviembre 2013. [En línea]. [Último acceso: 2017].

[19] R. R. De Adana Perez, «Diseño de la calidad a través de QFD,» 2011. [En línea]. Available: http://ricardoruizdeadana.blogspot.com/2011/05/qfduna-herramienta-para-alinear.html. [Último acceso: 2017].

[20] K. Schwaber, Agile Project Management with Scrum, 2014.

[21] J. M. Wargo, Apache Cordova 3 Programming, Pearson Education, Inc, 2014.

[22] The Apache Software Foundation, «Welcome to Apache Maven,» 0404 2016. [En línea]. Available: https://maven.apache.org. [Último acceso: 0604 2016]. [23] Drifty Co., «Company About - Ionic Platform,» 2012. [En línea]. Available: http://ionic.io/about. [Último acceso: 1004 2016].
[24] W3Schools, «The World's Largest Web Debeloper Site,» 2015. [En línea]. Available: http://www.w3schools.com/angular/angular_intro.asp. [Último acceso: 1004 2016].

[25] JBoss Developer, «JBoss Technology,» 0101 2014. [En línea]. Available: http://www.jboss.org/technology/. [Último acceso: 20 04 2016].

[26] RichFaces JBoss Community, «RichFaces, the JFS component,» 0101 2012. [En línea]. Available: https://developer.jboss.org/wiki/richfaceswikihomepag e. [Último acceso: 2004 2016].

[27] Mapzen, «Mapzen - an open, sustainable, and accessible mapping platform,» 0101 2015. [En línea]. Available: https://mapzen.com/. [Último acceso: 2004 2016].

[28] Leaflet Maps, «Leaflet, a JavaScript library for interactive maps,» 0101 2015. [En línea]. Available: http://leafletjs.com/. [Último acceso: 2004 2016].

[29] Oracle, «Java SE Documentation,» 2016. [En línea].

Available:

http://docs.oracle.com/javase/7/docs/. [Último acceso: 2004 2016].

[30] K. Farfán, Interviewee, Planes para el fortalecimiento turístico de Azogues. [Entrevista]. 13 022016 